\title{
Tunnel Surrounding Rock Displacement Prediction Using Support Vector Machine
}

\author{
BAO-ZHEN YAO
}

School of Civil Engineering \& Architecture, Beijing Jiaotong University, Beijing, 100044, P.R.China

CHENG-YONG YANG

School of Civil Engineering \& Architecture, Beijing Jiaotong University, Beijing, 100044, P.R.China

\author{
JIN-BAO YAO
}

School of Civil Engineering \& Architecture, Beijing Jiaotong University, Beijing, 100044, P.R.China

\author{
JIAN SUN
}

School of Transportation Engineering, Tongji University, Shanghai, 200092, P.R.China

Corresponding author Email: sunjian@tongji.edu.cn

Received: 26-05-2010; Accepted: 28-09-2010

\begin{abstract}
Multi-step-ahead prediction of tunnel surrounding rock displacement is an effective way to ensure the safe and economical construction of tunnels. This paper presents a multi-step-ahead prediction model, which is based on support vector machine (SVM), for tunnel surrounding rock displacement prediction. To improve the training efficiency of SVM, shuffled complex evolution algorithm (SCE-UA) is also performed through some exponential transformation. The data from the Chijiangchong tunnel are used to examine the performance of the prediction model. Results show that SVM is generally better than artificial neural network (ANN). This indicates that SVM is a feasible and effective multi-step method for tunnel surrounding rock displacement prediction.
\end{abstract}

Keywords: Prediction; Tunnel; Surrounding Rock Displacement; SVM; SCE-UA; Machine Learning

\section{INTRODUCTION}

Many problems, such as tunnel surrounding rock deformation and tunnel lining crack, may lead to the instability of the tunnel during tunnel construction. It is important to obtain reliable estimates of potential tunneling problems as early as possible. Statistical Process Control is an essential element for quality assurance ${ }^{1}$. The displacement statistics and analysis of the tunnel surrounding rock is the most common way to estimate the conditions of the tunnel. The lack of effective displacement prediction may result in increased cost and delays. Moreover, based on the displacement of tunnel surrounding rock, project managers identify the tunnel conditions and effectively operate their constructed facilities, for example, the appropriateness of support quantity and type can be determined. Furthermore, some potential danger occasions can be avoided by necessary emergency measures in 


\section{B.-Z. Yao et al.}

advance. There are many literatures devoted to the displacement prediction of tunnel surrounding rock $^{2-3}$.

For many engineering application, it is required that time series values be predicted many time-steps into the future. Many researches have applied multi-step-ahead (MS) techniques to deal with their problems. Cheng et al. ${ }^{4}$ proposed a multi-step-ahead prediction model to predict a long-term discharge in the hydropower system. In the model, dynamic spline interpolation with multilayer adaptive time-delay neural network had improved the prediction accuracy. Parlos et al. ${ }^{5}$ presented multi-step-ahead predictions for highly complex systems, and then they attempted to improve the prediction accuracy with dynamic recurrent neural networks. Lee and Billings ${ }^{6}$ presented multi-step ahead predictions for solving the non-linear time series examples. Chevillon and Hendry $^{7}$ used non-parametric direct multi-step-ahead to predict economic processes, particularly for a non-stationary data generating process. Other researches for multi-step-ahead can be found in the studies by Liu et al. ${ }^{8}$ and $\mathrm{Yu}$ et al. ${ }^{9}$

It is also essential to provide a dependable displacement of tunnel surrounding rock for planning, and management activities during tunnel construction. However, there are some changes in the ground conditions during tunnel construction, and thus it is very difficult to predict the displacement of tunnel surrounding rock accurately. Support vector machines (SVM) is a relatively new kind of learning machine which has been applied successfully in solving various time series forecasting problems. ${ }^{10-11}$ Other literatures on SVM can also be found in the studies by $\mathrm{Xu}$ et $a l^{12}$ and Reyaz-Ahmed et $a .^{13}$ Their numerical results indicate that SVM shows much resistance to the overfitting problem and can provide a high generalization performance.

These successful applications suggest that SVM is an acceptable tool to provide accurate displacement prediction of tunnel surrounding rock. However, the performance of SVM highly relies on the parameters and there is a need to determine the values of parameters. Many literatures have proved that the proper choice of the parameters in SVM will greatly affect its performance. Improper parameter values could result in the overfitting or underfitting of the training data points. ${ }^{14}$ To optimize the parameters in SVM, grid-search $^{15-16}$ is the most reliable method which tries values of each parameter across the specified search range using geometric steps. However, grid searcher requires too many evaluations at many points within the grid for each parameter. Quick and effective tools have been paid more attentions to improving the efficiency of the parameter optimization in SVM. Lorena et al. ${ }^{17}$ proposed genetic algorithms to select the proper parameter values for SVM. Ohn et al. ${ }^{18}$ presented a new kernel function to determine appropriate parameters in SVM. Hou and $\mathrm{Li}^{19}$ identified the parameters in SVM by using evolution strategy with covariance matrix adaptation. Lin et al. ${ }^{20}$ presented SVM for hydrological prediction and a shuffled complex evolution algorithm (SCE-UA) was used to identify appropriate parameters in SVM. Thus, this paper applies SCE-UA to find the appropriate parameters for SVM.

This paper is organized as follows. In Section 2, we describe the MS prediction problem on the displacement of tunnel surrounding. In Section 3 we introduce the SVM model for regression, and parameters optimization with SCE-UA. In Section 4, some computational results are discussed and lastly, the conclusions are provided in section 5 .

\section{MODEL DEVELOPMENTS}

The properties of the displacement prediction of tunnel surrounding with MS techniques not only depend on the observation values but also on the previous prediction. Thus, the recursive relation between inputs and outputs in MS prediction can be defined as the following:

$$
\begin{cases}\hat{x}_{t+p}=F\left(x_{t+p-m}, \cdots, x_{t}, \hat{x}_{t+1}, \cdots, \hat{x}_{t+p-2}, \hat{x}_{t+p-1}\right) & p<m \\ \hat{x}_{t+p}=F\left(\hat{x}_{t+p-m}, \cdots, \hat{x}_{t+p-2}, \hat{x}_{t+p-1}\right) & p \geq m\end{cases}
$$

Where $p$ is the number of steps ahead of $p$-step-ahead prediction model $F(\cdot)$ ( the horizon of $M S$ prediction ), $m$ is defined as the number of the inputs, $\hat{x}_{t+p}$ which has a "hat" represents an estimate of the output at time-step $t^{+} p$ and $x_{t+p-m}$ without a "hat" represents an observation. Obviously, if $p<m$, the model inputs consists of observation and prediction values, and if $p \geq m$, it consists of all prediction 
values. The structure of MS technique can be described in Fig.1.

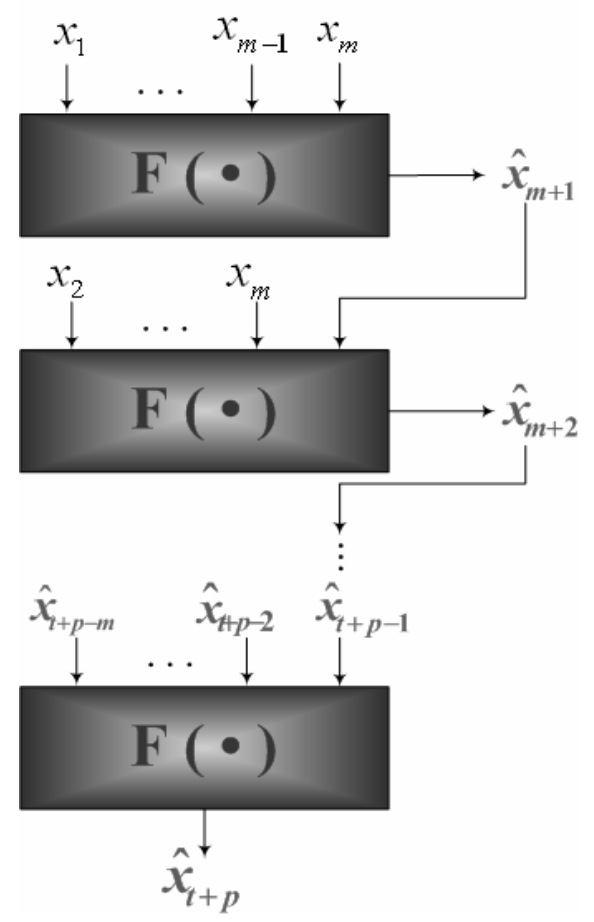

Fig.1 Structure of MS technique

The model is recursively used to predict time series values of many timesteps into the future. Assume the inputs of the current prediction are $\left(x_{t+p-m}, \cdots, x_{t+p-1}\right)$, the output is $\hat{x}_{t+p}$. Considering $m$ observations, some values of the inputs cannot be acquired directly from the observations but from the outputs of previous predictions. When $p<m$, the model inputs consist of observation and prediction values, the inputs should be $\left(x_{t+p-m}, \cdots, x_{t}, \hat{x}_{t+1}, \cdots, \hat{x}_{t+p-1}\right)$. When $p \geq m$, all the inputs come from the outputs of previous predictions, the inputs should be $\left(\hat{x}_{t+p-m}, \cdots, \hat{x}_{t+p-2}, \hat{x}_{t+p-1}\right)$. In the same way, the prediction process continues until all the prediction values are attained. For example, if let $m=5$, it means that the number of inputs ( in this study, it is also the number of observations ) is 5. $p$ is the number of steps which will increase with the prediction process. At first, $X=\left[x_{1}, x_{2}, x_{3}, x_{4}\right.$, $x_{5}$ ] as input variables are used to predict $x_{6}$ (now $p=1$ ). Then, $X=\left[x_{2}, x_{3}, x_{4}, x_{5}, x_{6}\right]$ are selected as input variables to predict $x_{7}$ (now $p=2$ ). Since the value of $x_{6}$ is not from observations but the output of the preceding prediction, the inputs for predicting $x_{7}$ should be $X=\left[x_{2}, x_{3}, x_{4}, x_{5}, \hat{x}_{6}\right]$. Similarly, when predicting $x_{11}$ (now $p=6>m$ ), the inputs are from $x_{6}$ to $x_{10}$ and the five values are all from the previous prediction. Thus, the input variables for predicting $x_{11}$ are $X=\left[\hat{x}_{6}, \hat{x}_{7}, \hat{x}_{8}, \hat{x}_{9}, \hat{x}_{10}\right]$. In the same way, $\hat{x}_{12}, \cdots, \hat{x}_{t+p}$ can be obtained by MS technique.

\section{SVM FOR TUNNEL SURROUNDING ROCK DISPLACEMENT PREDICTION}

SVM is a learning machine which shows high generalization ability by using a set of high dimensional linear functions, and thus it can capture reliability data patterns more easily than other models.

\subsection{SVM for regression}

Given a data $\operatorname{set}\left\{x_{k}, y_{k}\right\}, k=1,2, \ldots s \quad x_{k} \in R^{m}$ is the input vector and $y_{k} \in R^{n}$ is the desire value. SVM estimates the function by the following function:

$$
f(x)=<w, x>+b, \quad w, x \in R^{m}, b \in R^{n}
$$

Here, $\langle w, x\rangle$ is the feature of the inputs. The coefficients $w$ and $b$ are estimated by minimizing the regularized risk function.

$$
\text { MinJ }=\frac{1}{2}\|w\|^{2}+C \cdot R_{\text {emp }}[f]
$$




\section{B.-Z. Yao et al.}

The first term $\frac{1}{2}\|w\|^{2}$ is called the regularized term which is used as a measurement of function flatness. The second term $R_{\text {emp }}[f]$ is the so-called loss function to measuring the empirical error. $C$ is a regularization constant determining the trade-off between the training error and the generalization performance.

Because the cost function for building the model ignores any training data close to the model prediction (within a threshold $\varepsilon$ ), introduction of relaxation variables $\xi$, $\xi^{*}$ is used to cope with infeasible constraints of the optimization problem. To get the estimation of $w$ and $b$, the Eq.(3) can be transformed to the following function (4)

$$
\begin{aligned}
& \operatorname{Min} J=\frac{1}{2}\|w\|^{2}+C \sum_{i=1}^{s}\left(\xi_{i}^{*}+\xi_{i}\right) \\
& \text { s.t. }\left\{\begin{array}{c}
y_{i}-<w, x_{i}>-b \leq \varepsilon+\xi_{i}^{*} \\
<w, x_{i}>+b-y_{i} \leq \varepsilon+\xi_{i} \\
\xi_{i}^{*}, \xi_{i} \geq 0
\end{array}\right. \\
& \text { Let } w=\sum_{i=1}^{s}\left(\alpha_{i}-\alpha_{i}^{*}\right) x_{i}
\end{aligned}
$$

Thus, $f(x)=\sum_{i=1}^{s}\left(\alpha_{i}-\alpha_{i}^{*}\right)<x_{i}, x_{j}>+b$

By introducing kernel function $K\left(x_{i}, x_{j}\right)$ the Eq.(6) can be rewritten as follows:

$$
f(x)=\sum_{i=1}^{s}\left(\alpha_{i}-\alpha_{i}^{*}\right) K\left(x_{i}, x_{j}\right)+b
$$

where $K\left(x_{i}, x_{j}\right)$ is the so-called kernel function which is proven to simplify the use of a mapping. The value of $K\left(x_{i}\right.$, $x_{j}$ ) is equal to the inner product of two vectors. $x_{i}$ and $x_{j}$ in the feature space $\varphi\left(x_{i}\right)$ and $\varphi\left(x_{j}\right)$, that is, $K\left(x_{i}, x_{j}\right)=$ $\varphi\left(x_{i}\right) \bullet \varphi\left(x_{j}\right)$. By the use of kernels, all necessary computations can be performed directly in input space, without having to compute the map $\varphi(x)$. For more details can be seen in the studies by Vapnik ${ }^{11}$ and Cao et al $^{21}$.

\subsection{Applying SVM in tunnel surrounding rock displacement prediction}

Since the rock mass is heterogeneous and the advance deformation rate is not a constant, the displacement prediction of tunnel surrounding rock is a difficult task. We attempt to use MS techniques to estimate the future displacements with the historical data. Referring to previous literatures, ${ }^{22}$ it is feasible that the number of the inputs is set to 5. Here, we apply the displacement of the first five data to predict the displacement of the $6^{\text {th }}$ data. Then the data (from the $2^{\text {nd }}$ data to the $6^{\text {th }}$ prediction value) are used as input to predict the $7^{\text {th }}$ data. The rest can be done in the same manner. The prediction process of MS based on SVM can be described in Fig 2.

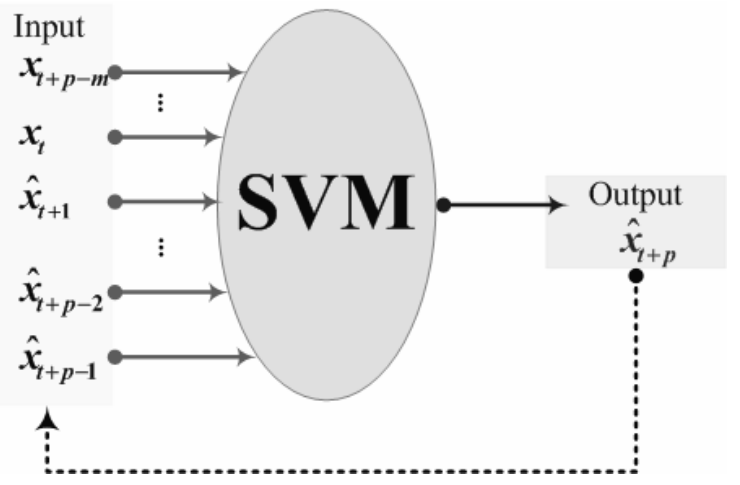

Fig. 2 The prediction process of MS based on SVM

\subsection{SCE-UA for parameters optimization in SVM}

It is appropriate to apply SVM for predicting the tunnel surrounding rock displacement. However, there are two key factors which will directly affect the ability of SVM. One is the selection of the kernel function; the other is the identification of the parameters $C, \varepsilon$ and $\sigma$. Most of the previous researches suggested that RBF kernel function was reasonable for SVM for regression. In this study, SCE-UA algorithm ${ }^{23}$ is applied to optimize the parameter $(C, \varepsilon$ and $\sigma)$ 
in SVM.

The SCE-UA algorithm has been successfully used in the area of surface and sub-surface hydrology for the calibration of rainfall-runoff models and identification of parameters of aquifer formation ${ }^{24}$. The SCE-UA algorithm combines the strengths of the simplex procedure of Nelder and Mead ${ }^{25}$ with: 1) the concept of controlled random search after work of Price ${ }^{26}$; 2) competitive evolution after Holland ${ }^{27}$; and 3) the concept of complex shuffling ${ }^{23,28}$. The synthesis of these three concepts makes the SCE-UA algorithm not only effective and robust but also flexible and efficient.

A general description of the steps of the SCE-UA algorithm is given below (more detailed information can be found in the studies by Duan et al. ${ }^{23,28}$ and Nunoo et al. ${ }^{29}$ ).

Step 1 Select $s$ points randomly from the feasible solution space;

Step 2 Sort the $s$ points in increasing order such that the first point represents the smallest function value.

Step 3 Partition the $s$ points into $p$ complexes, each containing $v$ points. The complexes are partitioned such that the first complex contains every $p(j-1)+1$ ranked point, the $h^{\text {th }}$ complex contains every $p(j-1)+h$ ranked point, and so on, where $j=1,2, \ldots$ $h \ldots v$.

Step 4 Evolve the complexes with the competitive complex evolution (CCE) algorithm (which will be elaborated later).

Step 5 Combine the points in all evolved complex into a single sample population; Sort the population in increasing order and shuffle (i.e. re-partition) them into $p$ complexes according to procedure specified in Step 3.

Step 6 If convergence criteria are satisfied stop the calculation, otherwise, continue.

Step 7 If the minimum number of complexes required in the population $p_{\text {min }}$ is less than $p$, remove the smallest complex and set $p=p-1, s=p v$ and return to Step 4 . If $p_{\min }=p$, return to Step 4 without reducing population size $p$.

One key component in the SCE-UA is the CCE algorithm. The CCE procedure employs the simplex downhill search method of Nelder and $\mathrm{Mead}^{25}$ in the generation of the offspring. The algorithm is summarized from the works ${ }^{23,28,29}$ as follows.
Step 1 Construct a sub-complex by randomly selecting $q$ points from the complex according to a trapezoidal probability distribution. The probability distribution is specified such that the better point has the higher chance of being chosen.

Step 2 Identify the worst point of the sub-complex and compute the centroid of the sub-complex by excluding the worst point.

Step 3 Attempt a reflection step by reflecting the worst point through the centroid (see Fig.3). If the newly generated point is within the feasible space, go to Step 4, otherwise, randomly generate a point within the feasible space and go to Step 6 .

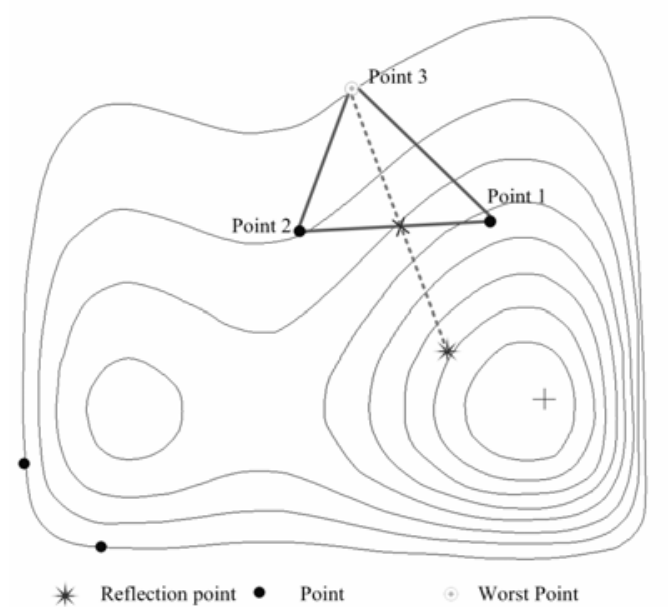

Fig. 3An example of reflection point

Step 4 If the newly generated point is better than the worst point, replace it with the new point and then go to Step 7, otherwise, go to Step 6.

Step 5 Attempt a contraction step by computing a halfway point between the centroid and the worst point (see Fig.4). If the contracted point is better than the worst point, replace it with the contraction point and go to Step 7. Otherwise, go to Step 6. 


\section{B.-Z. Yao et al.}

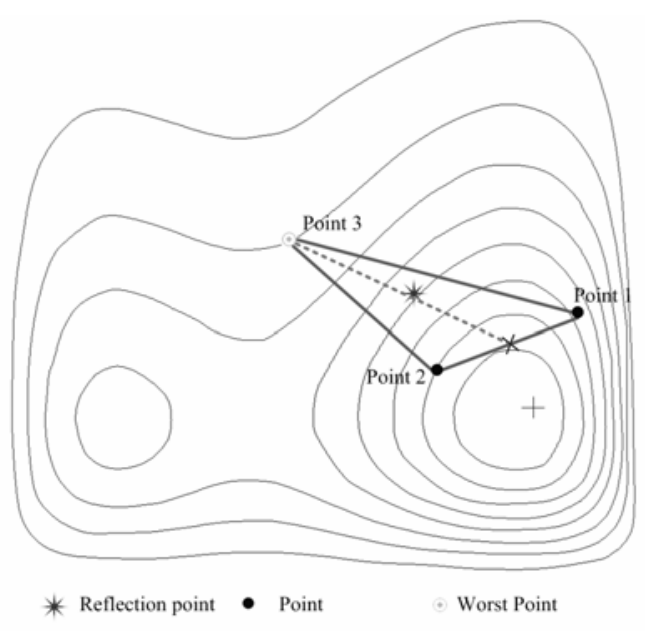

Fig. 4 An example of contracted point

Step 6 Randomly generate a point within the feasible space. Replace the worst point by the new point.

Step 7 Repeat Steps 2-6 $\alpha$ times, where $\alpha \geq 1$ is the number of consecutive offspring generated by the same sub-complex.

Step 8 Repeat Steps $1-7 \beta$ times, where $\beta \geq 1$ is the number of evolution steps taken by each complex before complexes are shuffled.

\section{CASE STUDY}

The Chijiangchong tunnel of the Wuhan-Guangzhou railway, which is a high-speed rail line connecting Wuhan city with Guangzhou city in China, is chosen as the study site. The length of the tunnel is about $385 \mathrm{~m}$ and its location is from DK1659+720 to DK1660+105 of the Wuhan-Guangzhou railway. We have chosen three sections, which are uniformly distributed throughout the tunnel, to acquire the data on the tunnel surrounding rock displacement. In general, the measurement frequency should be once per day at the beginning of the experiment and the frequency may be once every other day later. ${ }^{30}$ It is due to that the deformation rate in the beginning of the experiments is obviously more than one in the later. Thus, in this paper, the measurement frequency is one every day in the beginning thirteen days. Then the frequency is once every other day after the thirteenth day. The experiment continued until the tunnel surrounding rock displacement is almost stable. Here, we took the difference of two consecutive measurements < $0.1 \mathrm{~mm}$ as the termination condition. Thus, we acquired three sets of data and each set with thirty-two samples from the experiment from Sep 8 to Oct 28, 2007.

\subsection{Parameter Identification}

In this paper, the data is divided into training samples, testing samples and inspection samples according to the three sets. That is, the data from the first section and the third section are used for training and testing, the data from the second section are used for inspection. To reduce the search space, Due to RBF kernel function employed in SVM, previous literature ${ }^{15}$ suggested that the constraints of the three parameters were $C \in\left[2^{-5}, 2^{5}\right], \varepsilon \in\left[2^{-13}, 2^{-1}\right]$, and $\sigma \in[0,2]$. Then SVM is trained using SCE-UA algorithm. Here an objective function should be considered:

$$
\mathrm{RMSE}=\left[\frac{\sum_{i=1}^{n}\left(y_{i}-\hat{y}_{i}\right.}{n-p}\right]^{1 / 2}
$$

where $n$ is the number of testing samples, $p$ is the number of model parameters.

For the practical prediction model of the tunnel surrounding rock displacement, the three parameters values were selected through the SCE-UA algorithm. Fig. 5 shows the convergence of the calculation. Then, the optimal values are attained as $C=6.4371, \varepsilon=0.0032, \sigma=1.4129$.

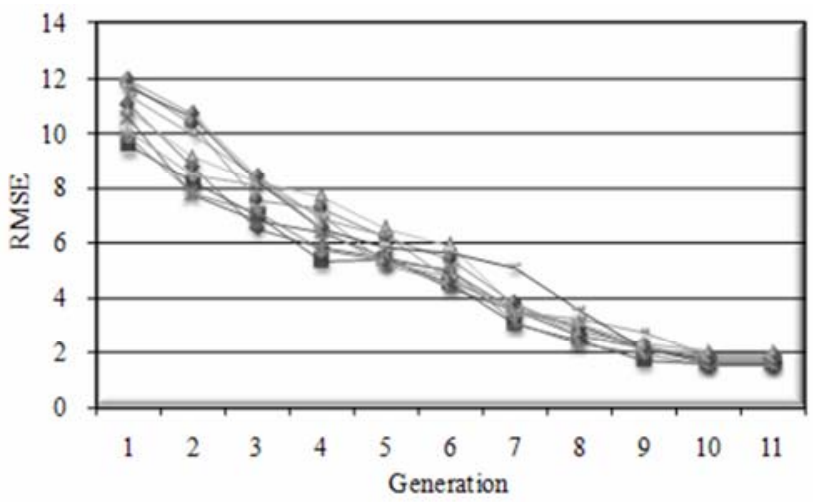

Fig. 5 The convergence of the calculations 


\subsection{The determination of the number of prediction steps}

The further prediction is very important for tunnel construction. However, the more steps there are, the poorer the prediction accuracies become. Due to the lack of measurements in input horizon, MS prediction technique needs the recursive use of single-step (SS) predictors for reaching the end-point. Thus, even small errors from preceding predictions are accumulated and propagated, then resulting in poor prediction accuracy in following predictions. To weight the number of prediction steps and accuracy, the data from the first section and the third section have been used to determine the number of prediction steps. Fig. 6 demonstrates the prediction errors of SVM under various prediction steps.

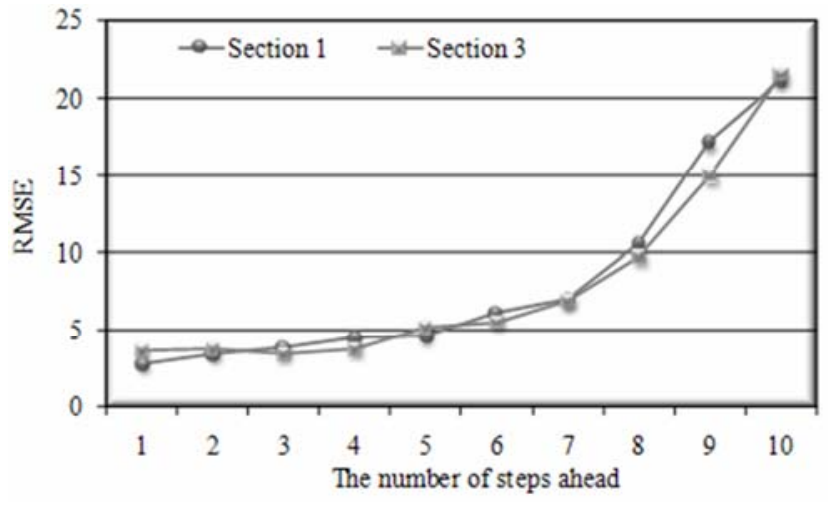

Fig. 6 Comparison of various prediction steps ahead

From Fig. 6, we can see there are both increasing trends of RMSEs of the displacement predictions on two sections as the increase of the step ahead. Especially, it can be observed that when the number of prediction steps is more than 7 , the prediction errors increase greatly. Therefore, the number of prediction steps ahead is determined as 7 in this study.

\subsection{Results}

To further analyze the characteristic of the MS prediction for the tunnel surrounding rock, we select the $6^{\text {th }}$ to the $12^{\text {th }}$ of the data from the second section as test bed I (note: the first five data as the inputs), and the $27^{\text {th }}$ to $33^{\text {rd }}$ of the data from the second section as test bed II. Here, the two test beds reflect respectively two typical cases, one is the phase that tunnel surrounding displacement increases obviously, and the other is the phase that tunnel surrounding displacement changes smoothly.

Then to evaluate the performance of the proposed model, a standard artificial neural network (ANN) model with three-layer is also introduced in this paper. To get a good comparison, the same input and output variables of ANN should be the same to the SVM. Then a scaled conjugate gradient algorithm ${ }^{31}$ is employed for training. To prevent overtraining and improve the generalization ability, the hidden neurons are generally optimized by a trial and error procedure. In this study, the final ANN architecture consists of five hidden neurons that yield the best performance. Then, we compare the performance of the SVM with that of ANN by using RMSE. Fig. 7 depicts the prediction performance of the two models on the two test beds. It can be found that two models obtain more accurate values at the former data than the latter data in each test bed. It is can be attributed to the fact MS predictor is based on the recursive use of SS predictor for reaching the end-point in the horizon. The prediction errors at the beginning of the horizon accumulate and propagate till to the end prediction. It is true that the prediction errors increase, however, in some real-world applications, especially for the displacement prediction of tunnel surrounding rock which has a relatively smaller period, moreover, it requires enough time for taking measures to combat the danger occasions. Thus it is acceptable to adapt to MS techniques to predict the future displacements.

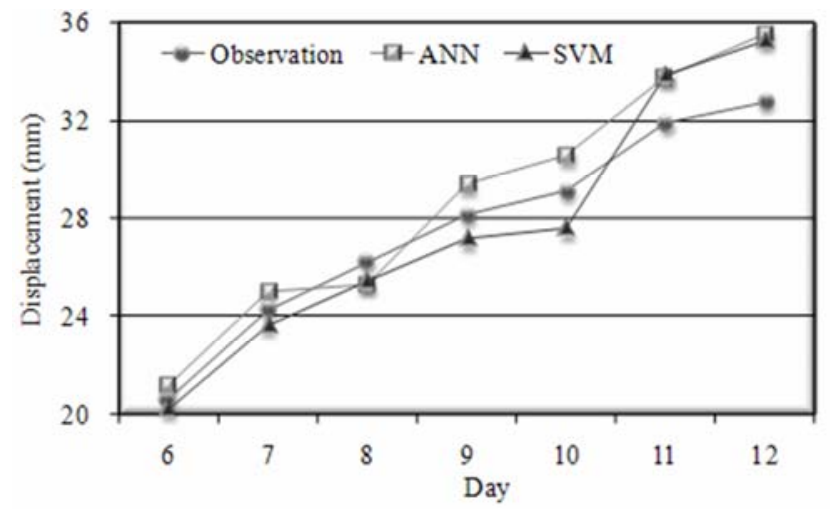

(a) Test bed I 


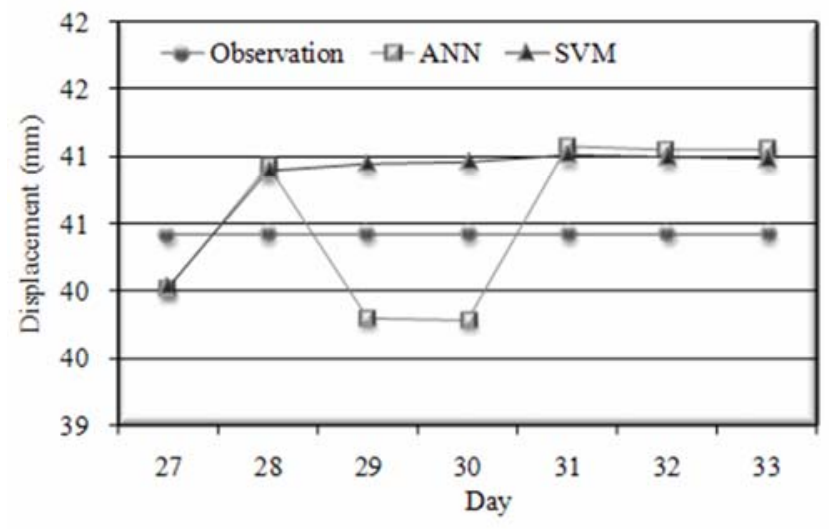

(b) Test bed II

Fig. 7 Comparison between the performances of SVM and ANN

The relationship between observations and predictions for the two test beds can be also illustrated in Fig. 8. It is obvious that the errors from SVM models generally are smaller than that of ANN. This can be explained that SVM uses the structural risk minimization principle to minimize the generalization error, while ANN uses the empirical risk minimization principle to minimize the training error. Furthermore, SVM always seeks to find the global solution while ANN may tend to fall into a local optimal solution. Therefore, it is feasible to solve the displacement prediction of tunnel surrounding rock with our model.

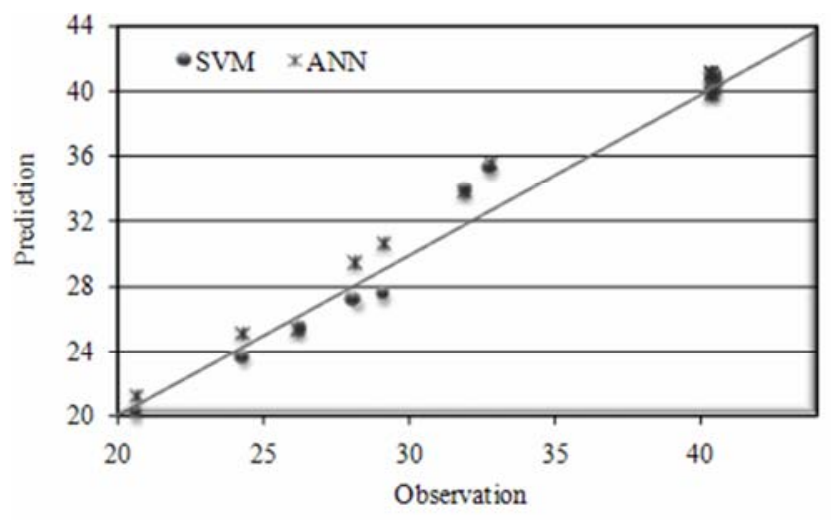

Fig. 8 Comparison between the prediction errors of SVM and ANN

\section{CONCLUSIONS}

Proving accurate displacement prediction in advance is one of the key aspects for identifying the potential danger occasions and reducing losses as possible during tunnel construction. If the long-time displacement prediction can be provided, there will be enough time for project managers to carry out some effective measures. Thus, the objective of this study is to develop a MS prediction model (SVM) for displacement prediction of surrounding rock. To improve the training efficiency of SVM, a SCE-UA algorithm is implemented for optimizing the parameters in SVM. Consider the fact that long-time prediction by MS technique will worsen the prediction errors. To determine the number of prediction steps, an experiment from the Chijiangchong tunnel is applied and the number of prediction steps is determined as 7 in this study. Then, the performance of the proposed method is also evaluated by the data. Results show that the proposed SVM model can provide better performance in most situations than ANN. Thus, SVM can be a potential tool for tunnel surrounding rock displacement prediction.

\section{Acknowledgments}

This research is financed by the National Natural Science Foundation of China through project 50978020 and the Excellent Doctor Innovation Research Funds of Beijing Jiaotong University through project 141065522.

\section{References}

1. McCoy, T., Yearout, R. and Patch, S. (2004); Misrepresenting quality data through incorrect statistical applications - A statistical quality control (SQC) case study. International Journal of Industrial Engineering-Theory Applications and Practice, 11(1):66-73.

2. Li, X.H., Zhao, Y., Jin, X.G., Lu, Y.Y., Wang, X.F.(2005); Application of Grey Majorized Model in Tunnel Surrounding Rock Displacement Forecasting. Lecture Notes in Computer Science, 3611:584-591.

3. Sellner P.J. (2000) Prediction of displacements in tunneling. 
Ph.D thesis, Graz University of Technology, Graz.

4. Cheng, C.T., Xie, J.X., Chau, K.W. and Layeqhifard, M. (2008); A new indirect multi-step-ahead prediction model for a long-term hydrologic prediction. Journal of Hydrology, 361(1-2):118-130.

5. Parlos, A.G., Rais, O.T. and Atiya, A.F. (2000); Multi-step-ahead prediction using dynamic recurrent neural networks. Neural Networks, 13(7):765-786.

6. Lee, K.L. and Billings, S.A. (2003); A new direct approach of computing multi-step ahead predictions for non-linear models. International Journal of Control, 76(8):810-822.

7. Chevillon, G. and Hendry, D.F.(2005); Non-parametric direct multi-step estimation for forecasting economic processes. INTERNATIONAL JOURNAL OF FORECASTING, 21(2):201-218.

8. Liu, J., Wang, W. and Golnaraghi, F. (2009); A multi-step predictor with a variable input pattern for system state forecasting. Mechanical Systems and Signal Processing, 23(5):1586-1599.

9. Yu. B., Yang, Z.Z. and Yu, B. (2009); Hybrid model for prediction of bus arrival times. Neural Network World, 19(3): 321-332.

10. Zhou, H., Li, W., Zhang, C. and Liu. J. (2009); Ice breakup forecast in the reach of the Yellow River: the support vector machines approach, Hydrology and Earth System Sciences Discussions, 6(2):3175-3198.

11. Cao, L.J. and Tay, F.E.H. (2003); Support Vector Machine with Adaptive Parameters in Financial Time Series Forecasting, IEEE Transactions on Neural Networks, 14(6):1506-1518.

12. Xu, X., Law, R. and Wu, T. (2009); Support Vector Machines with Manifold Learning and Probabilistic Space Projection for Tourist Expenditure Analysis. The International Journal of Computational Intelligence Systems, 2-1: 17-26.

13. Reyaz-Ahmed, A., Zhang, Y.Q. and Harrison, R.W.(2009); Granular Decision Tree and Evolutionary Neural SVM for Protein Secondary Structure Prediction. The International Journal of Computational Intelligence Systems, 2(4): 343-352.
14. Huang, C. L., \& Wang, C. J. (2006). A GA-based feature selection and parameters optimization for support vector machines. Expert Systems with Applications, 31, 231-240.

15. Hsu, C.W., Chang, C.C., and Lin, C.J. (2003); A Practical Guide to Support Vector Classification, Technical report, Department of Computer Science and Information Engineering, National Taiwan University.

16. Yu, B., Yang, Z..Z and Yao, B.Z. (2006); Bus arrival time prediction using support vector machines. Journal of Intelligent Transportation Systems, 10(4): 151-158.

17. Lorena, A.C. and de Carvalho, A.C.P.L.F. (2008); Evolutionary tuning of SVM parameter values in multiclass problems. NEUROCOMPUTING, 71(16-18): 3326-3334.

18. Ohn,S.Y., Nguyen, H.N., Chi, S.D.(2004); Evolutionary parameter estimation algorithm for combined kernel function in support vector machine. Lecture Notes in Computer Science, 3309:481-486.

19. Hou, S.M. and Li, Y.R. (2009); Short-term fault prediction based on support vector machines with parameter optimization by evolution strategy. EXPERT SYSTEMS WITH APPLICATIONS, 36(10): 12383-12391.

20. Lin, J.Y., Cheng, C.T., Chau, K.W.(2006); Using support vector machines for long-term discharge prediction, Hydrological Sciences Journal, 51(4): 599-612.

21. Vapnik, V.N. (1999); An Overview Of Statistical Learning Theory, IEEE Transactions on Neural Networks, 10(5): 988-999.

22. Xie, J. X., Cheng, C.T., Chau, K.W. and Pei, Y.Z. (2006); A hybrid adaptive time-delay neural network model for multi-step-ahead prediction of sunspot activity.International Journal of Environment and Pollution, 28(3-4):364-381.

23. Duan, Q. Y., Sorooshian, S. and Gupta, V. K. (1992). Effective and efficient global optimization for conceptual rainfall-runoff models. Water Resour. Res. 28(4): 1015-1031.

24. Duan, Q. Y., Sorooshian, S. and Gupta, V. K. (1994). Optimal use of the SCE-UA global optimization method for calibrating watershed models. J. Hydraul. Eng. 158(1): 265-284. 


\section{B.-Z. Yao et al.}

25. Nelder, J. A. and Mead, R. (1965). A simplex method for function minimization. Comput. J. 7(4): 308-313.

26. Price, W. L. (1987). Global optimization algorithm for a CAD workstation. J. Optim. Theory Appl. 55(1): 133-146.

27. Holland, J. H. (1975). Adaptation in Natural and Artificial Systems., University of Michigan Press, Ann Arbor, MI.

28. Duan, Q. Y., Gupta, V. K. and Sorooshian, S. (1993). Shuffled complex evolution approach for effective and efficient minimization. J. Optim. Theory Appl. 76(3): 501-521.
29. Nunoo, C., ASCE, M. and Mrawira, D. (2004). Shuffled Complex Evolution Algorithms in Infrastructure Works Programming. Journal of Computing In Civil Engineering 18(3): 257-266.

30. Schubert, W. and Steindorfer, A. Button, E.A.: (2002); Displacement Monitoring in Tunnels-an Overview. Felsbau 20(2):7-15.

31. Moller, M.F. (1993); A Scaled Conjugate Gradient Algorithm for Fast Supervised Learning, Neural Networks, 6(4): 523-533. 